\title{
Supernova Feedback on the Interstellar Medium and Star Formation
}

\author{
Gerhard Hensler \\ Institute of Astronomy, University of Vienna, Tuerkenschanzstr. 17, 1180 Vienna, Austria \\ email: gerhard.hensler@univie.ac.at
}

\begin{abstract}
Supernovae are the most energetic stellar events and influence the interstellar medium by their gasdynamics and energetics. By this, both also affect the star formation positively and negatively. In this paper, we review the development of the complexity of investigations aiming at understanding the interchange between supernovae and their released hot gas with the starforming molecular clouds. Commencing from analytical studies the paper advances to numerical models of supernova feedback from superbubble scales to galaxy structure. We also discuss parametrizations of star-formation and supernova-energy transfer efficiencies. Since evolutionary models from the interstellar medium to galaxies are numerous and apply multiple recipes of these parameters, only a representative selection of studies can be discussed here.
\end{abstract}

Keywords. stars: formation, ISM: kinematics and dynamics, ISM: bubbles, (ISM:) supernova remnants ISM: structure, galaxies: evolution, galaxies: ISM

\section{Introduction}

Since stars are formed within the coolest molecular material of the interstellar medium (ISM), the star-formation rate (SFR) should be determined simply by the gas reservoir and by the free-fall time $\tau_{\mathrm{ff}}$ of molecular clouds (Elmegreen 2002). This, however, raises a conflict between the ISM conditions and observed SFRs in the sense that $\tau_{\text {ff }}$ for a typical molecular cloud density of $100 \mathrm{~cm}^{-3}$ amounts to $10^{14} \mathrm{sec}$, i.e. $3 \cdot 10^{6}$ yrs. For the total galactic molecular mass of $10^{9}-10^{10} M_{\odot}$ the SFR should then amount to about 100 to $1000 M_{\odot} y r^{-1}$, what is by orders of magnitudes larger than observed and the gas reservoir within the Milky Way would have been used up today. This means, that the SF timescale must be stretched with respect to collapse or dynamical timescale by introducing a SF efficiency (SFE) $\epsilon_{\mathrm{SF}}$ and its definition could read: $\tau_{\mathrm{SF}}=\epsilon_{\mathrm{SF}}^{-1} \cdot \tau_{\mathrm{ff}}$.

Already in 1959 Schmidt argued that the SFR per unit area is related to the gas column density $\Sigma_{\mathrm{g}}$ by a power law with exponent $n$. Kennicutt (1998) derived from the $\mathrm{H} \alpha$ luminosity of spiral galaxies $\Sigma_{\mathrm{H} \alpha}$ a vertically integrated and azimuthally averaged SFR (in $M_{\odot} y r^{-1} p c^{-2}$ ), i.e. of gas disks in rotational equilibrium, and found a correlation with $n=1.4 \pm 0.15$ holding over more than 4 orders of magnitude in $\Sigma_{\mathrm{g}}$ with a drop below a density threshold at $10 M_{\odot} / p c^{2}$. While this relation establishes an equilibrium SFR, it is not surprising that the slope varies for dynamically triggered SF, as in starburst and merger galaxies and for high-z galaxies, when the disks form by gas infall, for the latter reaching $n=1.7 \pm 0.05$ (Bouché et al. 2007).

The ordinary Kennicutt-Schmidt (KS) relation can be understood by the simple analytical assumption allowing for the $\tau_{\mathrm{ff}}-\tau_{\mathrm{SF}}$ relation and for a uniform state of the ISM. Its equilibrium on disk scales requires that heating processes counteract to the natural cooling of plasmas. Besides the possible heating processes from dissipation of dynamical effects, as there are the differential rotation of the disk, gas infall, tidal interactions, shocks, etc., to the feedback by freshly produced stars, not for all of them it is obvious, 
how effectively they influence the SF by the expected self-regulation or vice versa trigger it.

Unfortunately, the issue of a general KS-law is confused by the similarity of slopes under various stellar feedback strengths (see e.g. sect. 2 in Hensler (2009)). Köppen, Theis, \& Hensler (1995) demonstrated already that the SFR achieves a dependence on $\rho_{\mathrm{g}}^{2}$, if the stellar heating is compensated by collisional-excited cooling radiation (e.g. Böhringer \& Hensler 1989). The coefficient of this relation determines $\tau_{\mathrm{SF}}$. Obviously, the SF in galaxies, therefore, depends on both the gas content and the energy budget of the ISM.

Since the most efficient stellar energy power is exerted by supernovae (SNe), and here particularly by the explosions of the shortly living massive stars as type II SNe, their feedback to the ISM is of fundamental relevance for the SF. In this paper, we overview and discuss the regulation of the ISM by the SF feedback thru SNe and more pronounced by their cumulative effect as superbubbles. Although the expression feedback of SNe also includes their release of freshly produced elements, here we only focus on the dynamical and energetic issues and refer the reader interested on the chemical evolutionary consequences to Hensler \& Recchi (2010).

\section{Supernova feedback}

\subsection{Energy release and the Interstellar Medium}

As SN explosions were since long known to expel vehemently expanding hot gas, Spitzer (1956) predicted this hot ISM phase to expand from the galactic disk where it cannot be bound or pressure confined to form a hot halo gas. Not before the 70's and with the aid of observations which made formerly unaccessible spectral ranges (from the FIR/submm to X-rays) available, the existence a hot gas component within our Milky Way was manifested and later also perceived in other disk galaxies. Although the ISM in its cool molecular component is conditioned for SF, hot gas regulates its dynamics as driver of shocks and turbulence as well as its energetics by heating thru cooling radiation and heat conduction, by this, exerting negative stellar feedback.

After these facts became internalized, a significant amount of mostly analytical explorations were dedicated to a first understanding of the ISM structure, its temporal behaviour with respect to the hot ISM (comprehensively reviewed by Spitzer (1990)), to volume filling factors and mass fractions of the gas phases. For this purpose, randomly distributed and temporally exploding SNe were considered with cooling and according expansion (Cioffi \& Shull 1991) and with transitions to/from the warm/cool gas (McKee \& Ostriker 1977) or as non-dynamically interchanging gas phases (see e.g. Habe, Ikeuchi, \& Takaka 1981, Ikeuchi \& Tomita 1983). In general, the action of SNe on the ISM is multifacetted (Chevalier 1977) and to a significant part affecting the SF. Because SNe type II happen on much shorter timescales than type Ia, all the ISM and SF feedback studies focus mostly on their energy deposit and dynamical issues.

While these latter studies include arbitrary SN rates with a description of the temporal size evolution of individual supernova remnants (SNRs), the SF dependence on the physical state is not yet properly treated. With a toy model consisting of 6 ISM components and at least 10 interchange processes Ikeuchi, Habe, \& Tanaka (1984) also included the formation of stars from giant molecular clouds. Those can only form from cool clouds which, on the other hand, are swept-up and condensed in SN shells, so that SF and SN explosions together with different gas phases and interchange processes form a consistent network. As a natural effect of this local consideration the SF can oscillates with the corresponding timescales. 
First dynamical approaches to the structure evolution of the ISM and gas disks, aiming at understanding the excitation of turbulence, were performed by Rosen \& Bregman (1995). As heating sources they took the energy of massive stellar winds only into account, which is at the lower range of stellar power to the ISM because of their very low energy transfer efficiencies (Hensler 2007). Nevertheless, their models already demonstrate the compression of gas filaments and the expulsion of gas vertically from the disk even with the inclusion of self-gravity. Indeed, the SF recipe is not self-consistent and the vertical boundary conditions seem to affect the vertical gas dynamics artificially.

In more recent numerical investigations of the ISM evolution in the context of SF and SN explosions Slyz et al. (2005) performed studies of the influence of SN feedback, self-gravity, and stars as an additional gravitational component on the SFR. The main issues can be summarized as that feedback enhances the ISM porosity, increases the gas velocity dispersion and the contrasts of $\mathrm{T}$ and $\rho$, so that smaller and more pronounced structures form. Since the SFR depends on most of these variables, more importantly, the SN feedback models reach SFRs by a factor of two higher than without feedback.

At the same time, de Avillez \& Breitschwerdt (2004) simulated the structure evolution of the solar vicinity in a box of $1 \times 1 \times 10 k p c^{3}$ size and identified the Local Bubble and its neighbouring Loop I in their models. In addition, filamentary neutral gas structures, resolved down to $0.625 \mathrm{pc}$, become visible and the vertical matter cycle. However, because of the lack of self-consistent SF they applied an analytical recipe of random SN events (de Avillez 2000) to their models that obviously underestimates the production of superbubbles from massive OB associations and, moreover, cannot reasonably achieve issues on the feedback of SNe on the SF. The ISM processes also do not include self-regulation effects by radiation or heat conduction, which lead to negative SF feedback.

\subsection{Star-formation triggering}

SN and stellar wind-driven bubbles sweep up surrounding gas, condense it, and are, by this, expected to excite SF in a self-propagating manner (abbreviated SPSF) as a positive feedback. In a cornerstone paper Gerola \& Seiden (1978) have demonstrated by a parameter study, how SNR expansion can lead to structure formation in galactic disks by SPSF. Their main aim, however, to reproduce the general spiral arm structure thru SPSF by means of the coherence of different timescales cannot be reached and most galaxies develop patchy structures randomly.

Such SF trigger by the condensation of swept-up gas in SN or more efficiently in superbubbles (see fig. 1) can be explored in detail by the investigation of the fragmentation timescale (see e.g. Ehlerova et al. 1997, Fukuda \& Hanawa 2000). Ehlerova et al. compared a self-similar analytical solution with the results of 3D numerical simulations of superbubble expansions in homogeneous media. The amount of energy supply from the final number of young stars in an $\mathrm{OB}$ association, the value of the sound speed, the stratification and density of the ambient medium, the galactic differential rotation, and the vertical gravitational force in the galactic disk, all these influence the fragmentation. The typical superbubble radius at which shells start to fragment decreases from almost $700 \mathrm{pc}$ at an ambient gas density $\mathrm{n}$ of $1 \mathrm{~cm}^{-3}$ to $200 \mathrm{pc}$ at $10 \mathrm{~cm}^{-3}$. While in thick disks like they exist in dwarf galaxies (DGs) nearly the whole shell fragments, in thin disks it is restricted to the galactic equator only. The SF process itself cannot be resolved in these studies and the assumption follows the line, that unstable fragments may become molecular and trigger the formation of molecular clouds in which new stars are formed. The main conclusion is that in DGs the SF may propagate in all directions possibly turning the system as the whole into a starburst, while in spiral galaxies the SF propagates within a thin strip near the symmetry plane only. Since the applied thin shell 


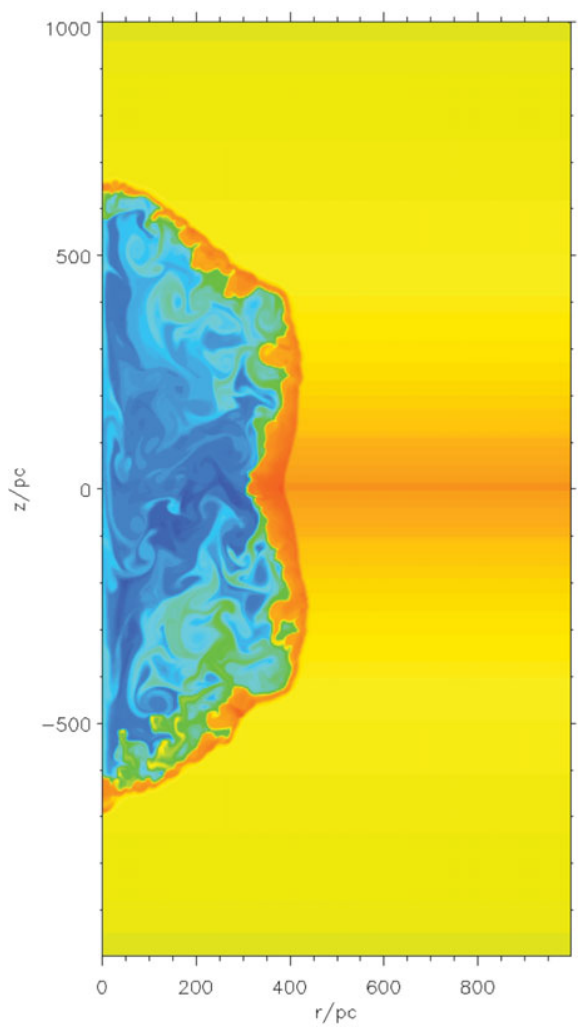

Figure 1. Density distribution of a superbubble after 20 Myrs. The superbubble results from 100 supernova typeII explosions at the origin of the coordinate system. The temporal sequence of explosions happens according to the lifetimes of stars in the mass range between 10 and 100 $M_{\odot}$ with a Salpeter IMF. The galactic disk is vertically composed of the three-phase interstellar medium, cool, warm and hot phase, respectively, with (central density $\rho_{0}\left[\right.$ in $\left.\mathrm{g} \mathrm{cm}^{-3}\right]$; temperature $\mathrm{T}[$ in $K]$; vertical scaleheight $\mathrm{H}[$ in $p c])$ of $\left(2 \times 10^{-24} ; 150 ; 100\right),\left(5 \times 10^{-25} ; 9000 ; 1000\right)$, $\left(1.7 \times 10^{-27} ; 2 \times 10^{6} ; 4000\right)$. The density varies from almost $10^{-23} \mathrm{~g} \mathrm{~cm}^{-3}$, in the densest part of the shell to $2 \times 10^{-28} \mathrm{~g} \mathrm{~cm}^{-3}$ in the darkest bubble interiors. (from Gudell 2002)

approximation is reasonably only a 0th-order approximation, in a recent paper Wünsch et al. (2010) (see also this volume) clarify that the shell thickness and the environmental pressure influences the fragments in the sense that their sizes become smaller for higher pressure. Nevertheless, the deviations from the thin-shell approximations are not large. Yet as a drawback the influences of magnetic fields is not taken into account.

The perception of SF trigger in SN or superbubble shells sounds reasonable from the point of view of numerical models because (as shown in fig. 1) sufficient ambient ISM mass is swept up, preferably in the gas disk itself, and is capable to cooling and gravitational instabilities. Shell-like distributions of young stars, are e.g. found in G54.4-0.3, called sharky (Junkes, Fürst, \& Reich 1992), in the Orion-Monoceros region (Wilson et al. 2005), more promising in the Orion-Eridanus shell (Lee \& Chen 2009), and in several superbubbles in the Large Magellanic Cloud, as e.g. Henize 206 (Gorjian et al. 2004). Also the formation of Gould's Belt stellar associations in the shell of a superbubble is most probable (Moreno, Alfaro, \& Franco 1999), however, still debated (Comeron \& Torra 1994).

Another possible feedback effect by $\mathrm{SN}$ is caused, when the ultra-fast SNR shock overruns a dense interstellar cloud, so that the clouds are quenched and stars are expected 
to be formed instantaneously. Although such cloud crushing is numerically modelled its effect on the necessary collapse of sub-clumps is not resolved simultaneously (Orlando et al. 2005).

Since in several DGs excessively high SFRs are observed (Hunter et al. 1998, van Zee, Skillman, \& Salzer 1998, Stil \& Isreal 2002 and further more), it is a matter of study whether starbursts are the result of a SF self-trigger mechanism or the issue of an external process because the objects are obviously linked to large enveloping gas reservoirs from which gas infall must be invoked (Mühle et al. 2005, Hensler et al. 2004). How the hot SN gas that transits from superbubbles to galactic winds interacts with infalling cold gas is yet unexplored but an attractive challenge from the present to the early universe (Recchi \& Hensler 2007). High-velocity clouds (HVCs) in our Milky Way on their passage through the hot halo gas are expected to be easily disrupted due to Kelvin-Helmholtz $(\mathrm{KH})$ instability. In contrast, model clouds of self-gravitating clouds including the effect of saturated heat conduction survive over almost 100 Myrs because they are mostly stabilized against KH instability (Vieser \& Hensler 2007). While their compression in the stratified halo gas should not be able to avoid SF, its absence in HVCs is still a mistery, so that its understanding would provide a further insight into the positive vs. negative feedback effect of SN gas on SF in clouds.

\subsection{Supernova energy impact}

Because of their enormous power in various forms, the majority of galaxy evolution models usually take the energy deposit of SNeII explosions into account as the only heating source for the ISM. Although it is generally agreed that the explosive energy of an individual SN lies around $10^{51} \mathrm{ergs}$ with significant uncertainties (or an intrinsic scatter), however, of one order of magnitude, the energy deposit as turbulent and consequently as thermal energy to the ISM is still more than unclear, but it is one of the most important ingredients for our understanding of galaxy formation (e.g. Efstathiou 2000, Silk 2003).

As a similar study, the energy release of massive stars as radiation-driven and windblown HiI regions can be considered. Analytical estimates for purely radiative HiI regions yielded an energy transfer efficiency $\epsilon$ of the order of a few percent (Lasker 1967). Although the additional stellar wind power $L_{w}$ can be easily evaluated from models and observations, its fraction that is transferred into thermal and turbulent energy is not obvious from first principles. Transfer efficiencies for both radiative and kinetic energies remain much lower from detailed numerical simulations than analytically derived and amount to only a few per mil (Hensler 2007, and references therein). Nevertheless, as valid for HII regions and because massive stars do not disperse from the SF site, SNeII explode within the stellar associations. While massive stars contribute significantly to the ISM structure formation as e.g. by cavities and holes, in the HI gas and chimneys of hot gas, on large scales the energy release by massive stars triggers the matter circulations via galactic outflows from a gaseous disk and galactic winds. By this, also the chemical evolution is affected thru the loss of metal-enriched gas from a galaxy (for observations see e.g. Martin, Kobulnicki \& Heckman (2002), for models e.g. Recchi \& Hensler (2006b)).

SN explosions as an immediate consequence of SF stir up the ISM by the expansion of hot bubbles, deposit turbulent energy into the ISM, thereby, heat the ISM and regulate the SF again (Hensler \& Rieschick 2002). This negative energy feedback is enhanced at low gravitation because the SN energy exceeds easily the galactic binding energy and drives a galactic wind. Although investigations have been performed for the heating (or energy transfer) efficiency $\epsilon_{\mathrm{SN}}$ of SNe (Thornton et al. 1998), superbubbles (e.g. Strickland et al. 2004), and starbursts (Melioli \& de Gouveia Dal Pino 2004) they are yet too simplistic and mostly spatially poorly resolved to account for quantitative results. 


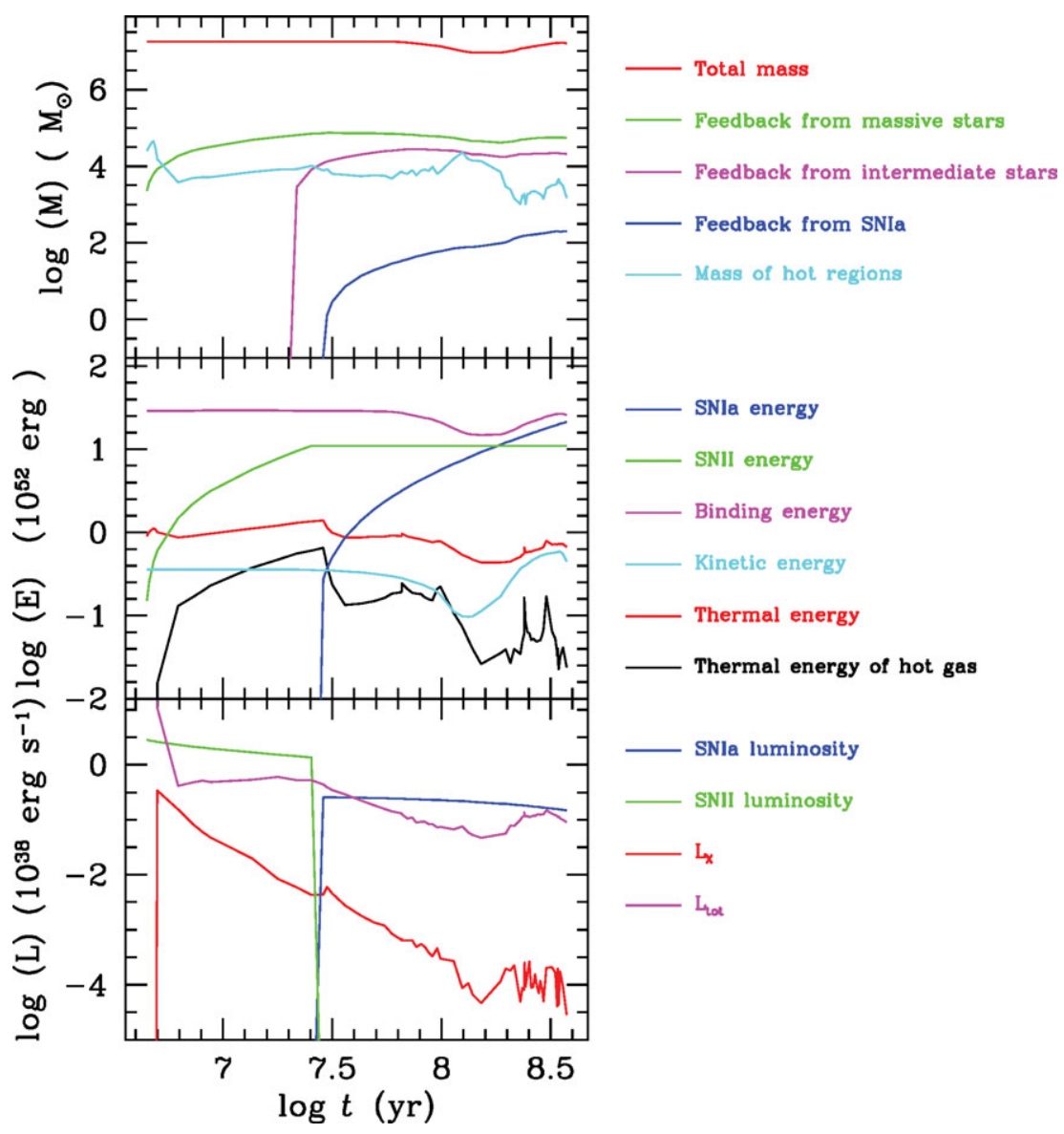

Figure 2. Temporal evolution of masses energies, and luminosities, respectively, of contained and released components for a single star-formation burst in a dwarf galaxy like I Zw 18 (Recchi et al. (2002). The green (light) curves refer to the supernovae typeII contribution. Comparison of the thermal energy (red line) and the accumulated supernova typeII energy (middle panel) shows that the thermal energy is almost not enhanced by SNeII.

Thornton et al. derived an efficiency $\epsilon_{\mathrm{SN}}$ of 0.1 from 1D SN simulations as already applied by chemo-dynamical galaxy models (Samland, Hensler \& Theis 1997), while unity is also used in some galaxy models (see sect. 3), but seems far too large.

Although numerical experiments of superbubbles and galactic winds are performed, yet they only demonstrate the destructive effect on the surrounding ISM but lack of selfconsistency and a complex treatment. Simulations of the chemical evolution of starburst DGs by Recchi et al. $(2002,2006)$, that are dedicated to reproduce the peculiar abundance patterns in these galaxies by different $\mathrm{SF}$ episodes, found, that $\epsilon_{\mathrm{SN}}$ can vary widely. A superbubble expanding from a stellar association embedded in a thick HI disk has, at first, to act against the surrounding medium, by this, is cooling due to its pressure work and radiation, but compresses the swept-up shell material and implies turbulent energy to the ISM. Here the superbubble expansion is efficiently hampered, but depends on the HI disk thickness (Recchi et al. 2009) and the energy loss by radiative cooling.

In fig. 2 the temporal behaviour of the above-mentioned starburst DG model by Recchi et al. (2002) is displayed. Comparison of the various kinds of energy contents reveal an heating efficiency $\epsilon_{\mathrm{SN}}$ of to about $18 \%$. While the accumulated SNII energy release reaches 
$10^{53}$ ergs after $20 \mathrm{Myrs}$, the thermal energy content starts at $10^{52}$ drops and increases successively but not above $1.8 \times 10^{52} \mathrm{ergs}$. For the subsequent SNIa explosions, always single events, the accumulated energy release is clearly discernible while the thermal energy decreases again and varies below $10^{52} \mathrm{ergs}$ so that here $\epsilon_{\mathrm{SN}} \rightarrow 0$. Moreover, if a closely following SF episode pushes its SNeII into the already existing chimney of a preceding superbubble, the hot gas can easily escape without any hindrance and thus affects the ISM energy budget much less. Recchi \& Hensler (2006a) found that depending on the external Hi density the chimneys do not close before a few hundred Myrs.

\section{Galaxy evolution}

Numerical simulations of galaxy evolution are only feasible with intermediate-scale spatial resolution so that SF cannot be resolved and must already be prescribed by reasonable recipes. Numerous papers have implemented SF criteria, such as e.g. threshold density $\rho_{\mathrm{SF}}$ with SF if $\rho_{\mathrm{g}} \geqslant \rho_{\mathrm{SF}}$, excess mass in a specified volume with respect to the Jeans mass, i.e. $\mathrm{M}_{\mathrm{g}} \geqslant M_{\mathrm{J}}$, convergence of gas flows $($ div $\cdot \underline{\mathbf{v}}<0)$, cooling timescale $t_{\text {cool }} \leqslant$ $t_{d y n}$, temperature limits $\mathrm{T}_{\mathrm{g}} \leqslant \mathrm{T}_{\text {lim }}$, Toomre's Q parameter, temperature dependent SFR, but to some extent also already under the assumption of the KS law (Dalla Vecchia \& Schaye 2008). This latter, however, is unjustified for non-equilibrium situations, because the SF should converge to this relation due to self-regulation. If at least some of these conditions are fulfilled, as a further step, the gas mass which is converted into stars, i.e. the SFE, has to be set e.g. by fixing an empirical value $\epsilon_{\mathrm{SF}}$ from observations, i.e. $\Delta m_{\mathrm{SF}}=\epsilon_{\mathrm{SF}} \cdot \rho_{\mathrm{g}} \cdot \Delta x^{3}$, where $\Delta x^{3}$ is e.g. the mesh volume in a grid code. If the numerical timestep $\Delta t$ is smaller than the dynamical timestep $\tau_{\mathrm{ff}}, \Delta m_{\mathrm{SF}}$ has to be weighted by this time ratio (Tasker \& Bryan 2006). Since $\epsilon_{\mathrm{SF}}$ must inherently depend on the local conditions so that it is high in bursting SF modes, as requested for the Globular Cluster formation, but of percentage level in the self-regulated SF mode, numerical simulations often try to derive the realistic SFE by comparing models of largely different $\epsilon_{\mathrm{SF}}$ with observations, as e.g. to reproduce gas structures in galaxy disks and galactic winds (e.g. $\epsilon_{\mathrm{SF}}=0.05$ and 0.5 in Tasker \& Bryan 2006, 2008). In addition, $\epsilon_{\mathrm{SN}}$ by them is fixed to $10^{51}$ erg per $55 M_{\odot}$ of formed stars $\left(1.8 \times 10^{49} \mathrm{erg} M_{\odot}^{-1}\right.$ by Dalla Vecchia \& Schaye 2008), so that these results can be treated indicatively but not yet quantitatively, since they also mismatch with the KS relation.

Theoretical studies by Elmegreen \& Efremov (1997) achieved a dependence of $\epsilon_{\mathrm{SF}}$ on the external pressure, while Köppen, Theis, \& Hensler (1995) explored a temperature dependence of the SFR. Furthermore, most galaxy evolutionary models at present lack of the appropriate representation of the different ISM phases allowing for their dynamics and their direct interactions by heat conduction, dynamical drag, and dynamical instabilities thru forming interfaces, not to mention resolving the turbulence cascade.

\section{Conclusions}

The dominating influence of SN explosions and superbubbles on structure, dynamics, and energy budget of the ISM are obvious and agreed. Signs and strengths of these feedback effects are, however, widely uncertain. Whether the feedback is positive (trigger) or negative (suppression) can be understood analyticly from first principles, but because of the non-linearity and the complexity level of the acting plasmaphysical processes clear results cannot be quantified reliably. In addition, the temporal behaviour varies by orders of magnitude because of the changing conditions. In summary, the energy transfer efficiencies of SNe and superbubbles to the ISM are much below unity and depend on the 
temporal and local conditions, but must not be overestimated. Spatially and temporarily resolved simulations of SN and superbubbles in an extended environment with varying conditions of the ISM are necessary in order to connect large-scale effects on SF clouds with the existing detailed simulations on the star-forming scales.

\section{Acknowledgements}

The author is grateful to Simone Recchi for substantial contributions to this topic and providing fig. 2. The attendance of the symposium was funded by the key programme "Computational Astrophysics" of the University of Vienna under project no. FS538001.

\section{References}

Böhringer, H. \& Hensler, G. 1989, A\&A, 215, 147

Bouché, N., Cresci, G., Davies, R., et al. 2007, ApJ, 671, 303

Chevalier, R.A. 1977, ARA\&A, 15, 175

Cioffi, D.F. \& Shull, J.M. 1991, ApJ, 367, 96

Comeron, F. \& Torra, J. 1994, A\&A, 281, 35

Dalla Vecchia, C. \& Schaye, J. 2008, MNRAS, 387, 1431

de Avillez, M.A. 2000, MNRAS, 315, 479

de Avillez, M.A. \& Breitschwerdt, D. 2004, A\&A, 425, 899

Efstathiou, G. 2000, MNRAS, 317, 697

Ehlerova, S., Palous, J., Theis, C., \& Hensler, G. 1997, A\&A, 328, 111

Elmegreen, B.G. 2002, ApJ, 577, 206

Elmegreen, B.G. \& Efremov, Y.N. 1997, ApJ, 480, 235

Fukuda, N. \& Hanawa, T. 2000, ApJ, 533, 911

Gerola, H. \& Seiden, P.E. 1978, ApJ, 223, 129

Gorjian, V., Werner, M.W., Mould, J.R., et al. 2004, ApJS, 154, 275

Gudell, A. 2002, diploma thesis, University of Kiel

Habe, A., Ikeuchi, S., \& Tanaka, Y.D. 1981, PASJ, 33, 23

Hensler, G. 2007, in eds. E. Ensellem et al., Chemodynamics: from first stars to local galaxies Proc. CRAL-Conference Series I, EAS Publ. Ser. No. 7, p. 113

Hensler, G. 2009, in: J. Andersen, J. Bland-Hawthorn, \& B. Nordstroem (eds.), The Galaxy Disk in Cosmological Context, Proc. IAU Symposium No. 254, p. 269

Hensler, G. \& Recchi, S. 2010, in: K. Cunha, M. Spite \& B. Barbuy, (eds.), Chemical Abundances in the Universe: Connecting First Stars to Planets, Proc. IAU Symp. No. 265, p. 325

Hensler, G. \& Rieschick, A. 2002, in: E. Grebel \& W. Brandner (eds.), Modes of Star Formation and the Origin of Field Populations, ASP Conf. Ser., 285, 341

Hensler, G., Köppen, J., Pflamm, J., \& Rieschick, A. 2004, in: P.-A. Duc, J. Braine, E. Brinks (eds.), Recycling intergalactic and interstellar matter, Proc. IAU Symp. No. 217, p. 178

Hunter, D.A., Wilcots, E.M., van Woerden, H., et al. 1998, ApJ, 495, L47

Kennicutt, R.J. 1998, ApJ, 498, 541

Ikeuchi, S. \& Tomita, H. 1983, PASJ, 35, 56

Ikeuchi, S., Habe, A., \& Tanaka, Y.D. 1984, MNRAS, 207, 909

Junkes, N., Fürst, E., \& Reich, W. 1992, A\&A, 261, 289

Köppen, J., Theis, C., \& Hensler, G. 1995, A\&A, 296, 99

Lasker, B.M. 1967, ApJ, 149, 23

Lee, H.-T. \& Chen, W.P. 2009, ApJ, 694, 1423

Martin, C.L., Kobulnicki, H.A., \& Heckman, T.M. 2002, ApJ, 574, 663

McKee, C.F. \& Ostriker, J.P. 1977, ApJ, 218, 148

Melioli, C. \& de Gouveia Dal Pino, E.M. 2004, A\& A, 424, 817

Moreno, E., Alfaro, E.J., \& Franco, J. 1999, ApJ, 522, 276

Mühle, S., Klein, U.,Wilcots, E. M., \& Hüttermeister, S. 2005, AJ, 130, 524

Orlando, S., Peres, G., Reale, F., et al. 2005, A\&A, 444, 505 
Recchi, S. \& Hensler, G. 2006a, A\& A, 445, L39

Recchi, S. \& Hensler, G. 2006b, Rev. Mod. Astronomy, 18, 164

Recchi, S. \& Hensler, G. 2007, A\& A, 476, 841

Recchi, S., Hensler, G., \& Anelli, D. 2009, arXiv:0901.1976

Recchi, S., Hensler, G., Angeretti, L., \& Matteucci, F. 2006, A\&A, 445, 875

Recchi, S., Matteucci, F., D'Ercole, A., \& Tosi, M. 2002, A\&A, 384, 799

Rosen, A. \& Bregman, J.N. 1995, ApJ, 440, 634

Samland, M., Hensler, G., \& Theis, C. 1997, ApJ, 476, 544

Schmidt, M. 1959, ApJ, 129, 243

Slyz, A.D., Devriendt, J.E.G., Bryan, G., \& Silk, J. 2005, MNRAS, 356, 737

Silk, J. 2003, MNRAS, 343, 249

Spitzer, L. 1956, ApJ, 124, 20

Spitzer, L. 1990, ARA\&A, 28, 71

Stil, J. M. \& Israel, F. P. 2002, A\&A, 392, 473

Strickland, D.K., Heckman, T.M., Colbert, E.J.M., et al. 2004, ApJ, 606, 829

Tasker, E.J. \& Bryan, G.L. 2006, ApJ, 641, 878

Tasker, E.J. \& Bryan, G.L. 2008, ApJ, 673, 810

Thornton, K., Gaudlitz, M., Janka, H.-Th., \& Steinmetz, M. 1998, ApJ, 500, 95

van Zee, L., Skillman, E.D., \& Salzer, J.J. 1998, AJ, 116, 1186

Vieser, W. \& Hensler, G. 2007, A\&A, 472, 141

Wilson, B.A., Dame, T.M., Masheder, M.R.W., \& Thaddeus, P. 2005, A\&A, 430, 523

Wünsch, R., Dale, J.E., Palous, J., \& Whitworth, A.P. 2010, MNRAS, in press 


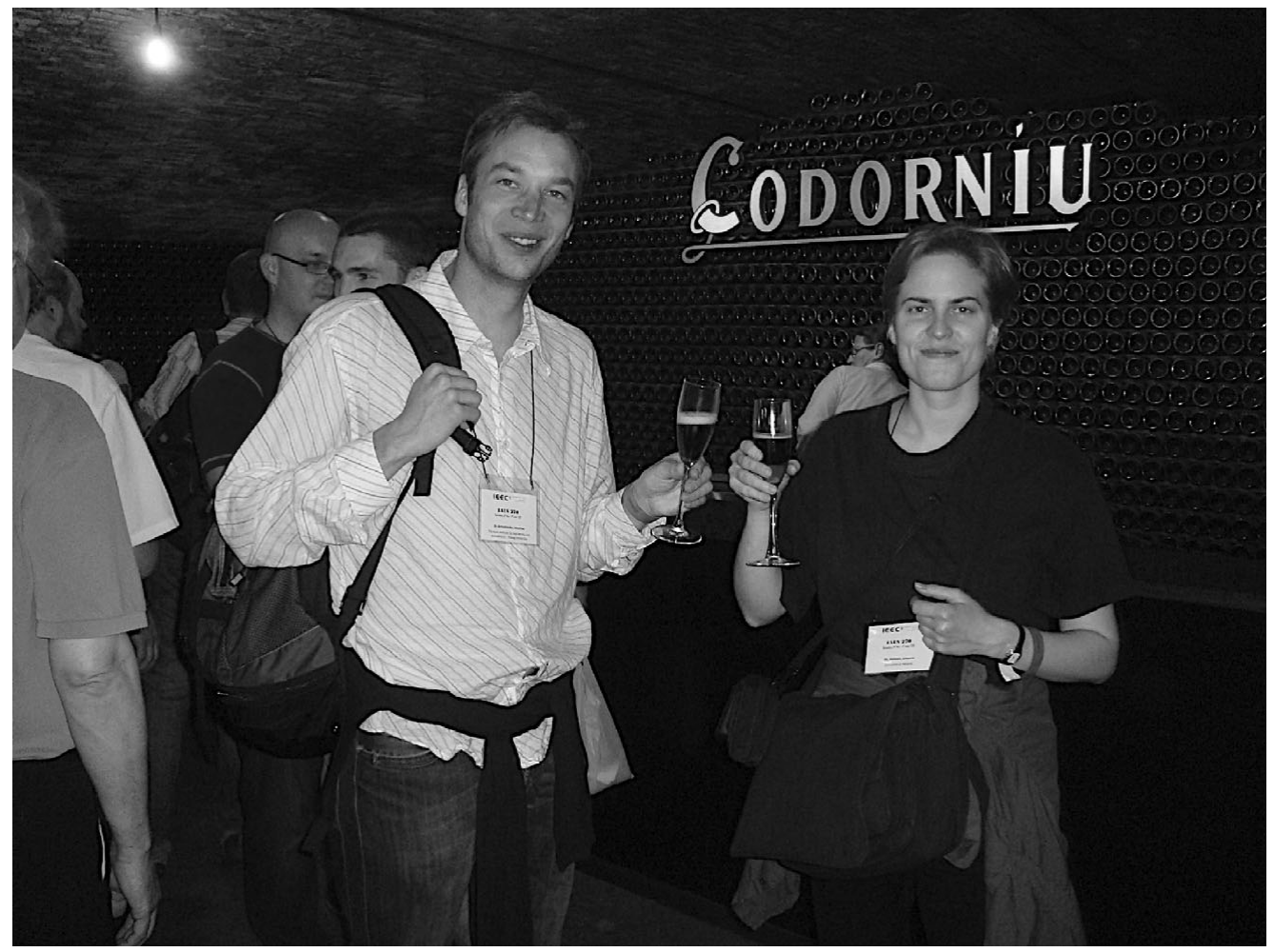

Matthias Gritschneder and Johanna Malinen 